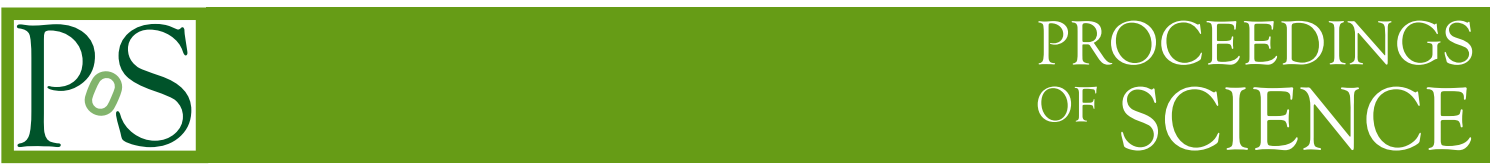

\title{
Operation and Performance of the CMS Silicon Tracker
}

\author{
Erik Butz ${ }^{* \dagger}$ \\ Massachussetts Institute of Technology \\ E-mail: ebutz@mit.edu
}

\begin{abstract}
The CMS silicon tracker is the largest silicon detector ever built. It consists of a hybrid pixel detector with 66 million channels and a $200 \mathrm{~m}^{2}$ silicon strip detector with 10 million readout channels. We describe the operation of this detector during the first three year of LHC both during proton-proton as well as heavy ion collisions with results on the operational performance, calibration, signal-to-noise ratio, timing, etc. The resolution and efficiency of the track and vertex reconstruction are measured with data and compared to the results from simulation. With increasing integrated luminosity, monitoring of radiation-induced effects becomes more and more important. Our methods for measuring the evolution of full depletion voltage and leakage current will be presented and the results discussed.
\end{abstract}

36th International Conference on High Energy Physics,

July 4-11, 2012

Melbourne, Australia

\footnotetext{
${ }^{*}$ Speaker.

† for the CMS collaboration
} 


\section{Introduction}

The CMS central tracking system [1] is composed of a silicon pixel and a silicon strip tracker. Together they provide high precision measurements of charged particle trajectories up to $|\eta|<2.5^{1}$.

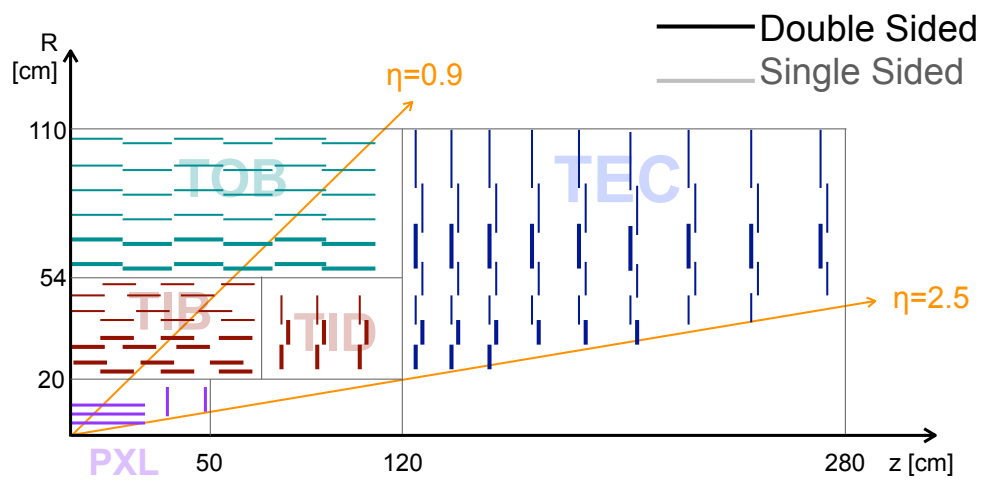

Figure 1: $r z$ view of one quarter of the CMS silicon tracker. For the silicon strip tracker, layers with so-called stereo modules (see text for details) are drawn as thick lines.

The CMS pixel tracker has an active silicon area of $1.1 \mathrm{~m}^{2}$, grouped into 1440 modules. These are arranged in three layers in the barrel region and two end cap disks on either side of the interaction point. The pixel detector uses $n$-in- $n$ silicon sensors with a total of 66 million pixels of $100 \times 150 \mu \mathrm{m}^{2}$ and a $285 \mu \mathrm{m}$ silicon bulk providing precise 2D measurements. The CMS silicon strip tracker is the largest of its kind ever built. Its active area is almost $200 \mathrm{~m}^{2}$ with 15148 individual silicon modules. These are arranged in 10 barrel layers and $3+9$ endcap disks. In the outer parts of the silicon tracker, two silicon sensors are daisy-chained to form the silicon module. The readout pitch ranges from $85 \mu \mathrm{m}$ in the inner layers of the inner barrel to $205 \mu \mathrm{m}$ in the outer rings of the end caps. The strip tracker uses $p$-in- $n$ silicon with 300(500) $\mu \mathrm{m}$ sensor thickness in the inner(outer) parts of the detector and a total of 9.6 million readout channels. In four layers (three rings) of the barrel (endcap) region, the strip tracker uses so-called stereo modules. These are modules in which two silicon sensors are mounted back-to-back with a $100 \mathrm{mrad}$ stereo angle to effectively provide a 2D hit resolution also in the strip tracker. An $r z$ view of one quarter of the CMS tracker is shown in figure 1. Both tracking sub-detectors of CMS are using an analog readout via optical links which retains information about the absolute signal height. The pixel detector employs an on-chip data sparsification to reduce the data volume to be shipped out. For the strip tracker all data processing is performed in the off-detector electronics.

The CMS silicon trackers have been in more or less continuous operation during the years 2010-12. The fraction of operational channels has been reasonably constant over this period, not changing by more than a few per cent. The current active detector fraction is about $97 \%$ for the pixel and $97.5 \%$ for the strip tracker. During 2012, CMS has been delivered a total of $6.65 \mathrm{fb}^{-1}$ of data of which $6.15 \mathrm{fb}^{-1}$ have been recorded (up to July 2012, cf. figure 2). This corresponds to a data taking efficiency of $92.5 \%$ which is higher than in 2011 despite the fact that the energy

\footnotetext{
${ }^{1} \mathrm{CMS}$ uses a right-handed coordinate system with $x$ pointing towards the center of the LHC ring, $y$ pointing upwards and $z$ pointing in the direction of the counter-clockwise beam. The azimuthal angle is denoted $\vartheta$, the polar angle as $\varphi$. The pseudorapidity is defined as $\eta=-\ln \tan (\vartheta / 2)$.
} 
of the LHC was increased and pile-up conditions became more challenging. In figure 3, the break down of the luminosity losses among the individual CMS sub-detectors is shown. The CMS silicon trackers were responsible for $29 \%$ of the lost luminosity. Of this, $16 \%$ were caused by the strip tracker, $12 \%$ by the pixel detector, and about $1 \%$ by failures of power supplies.

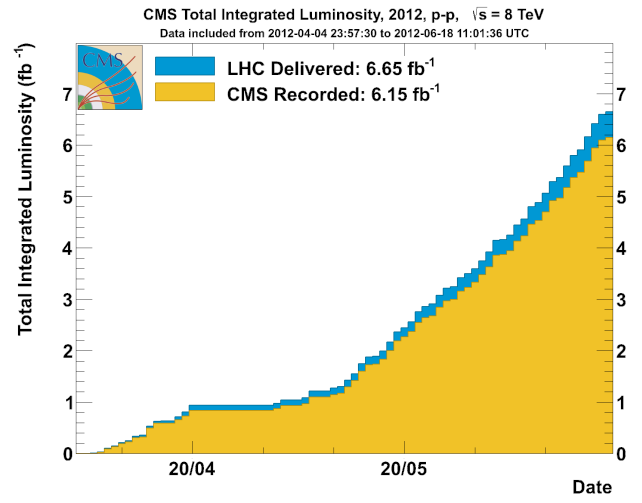

Figure 2: Delivered and recorded luminosity for CMS in 2012 (up to July 2012).

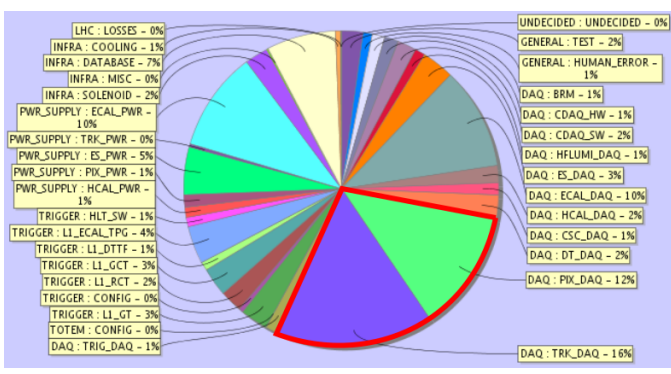

Figure 3: Contribution of lost luminosity split by CMS subsystem. The contribution by the tracking sub-detectors is highlighted in red.

The services of both silicon detectors have been performing very stably during the operational years 2011 and 2012. The power supply system [2] is running well and the exchange rate of power supply units remains well below $1 \%$ per year. The cooling plants are running stably with coolant temperatures of $+4^{\circ} \mathrm{C}$ for the strip tracker and $0^{\circ} \mathrm{C}$ for the pixel detector. For both detectors the operating temperature will be lowered after the Long Shutdown 1 of the LHC.

\section{Radiation monitoring}

During the two years 2011 and $2012^{2}$, the silicon detectors acquired a radiation dose corresponding to $13 \mathrm{fb}^{-1}$. The effects of irradiation are closely monitored by performing various scans of the radiation related quantities. For the depletion voltage two types of scans are performed regularly. One is using tracks from $p p$ collisions whereas the other relies on random triggers during periods without beam. At least once per month, a small scan of the bias voltage with $p p$ collisions is performed. For this, a set of representative power groups from different parts of the tracker is scanned.

For the leakage current, measurements are performed using both in-situ measurements from the detector control units (DCUs) on each module and current measurements from the power supply units. In the latter, the current measurement is integrated over several modules (3-12) due to the limited granularity of the power system. Both methods provide consistent results.

In figure 4, the leakage current measured by the DCUs, corrected for temperature effects, is compared to a FLUKA [3,4] simulation of the expected irradiation normalized to $1 \mathrm{MeV}$ neutron equivalent $/ \mathrm{cm}^{2}$. One can see that in general there is good agreement between the measured leakage current and the simulated flux. In figure 5, the measured leakage current is compared to a prediction. One can see that there is good agreement between the simulation and the data.

\footnotetext{
${ }^{2}$ up to July 2012
} 


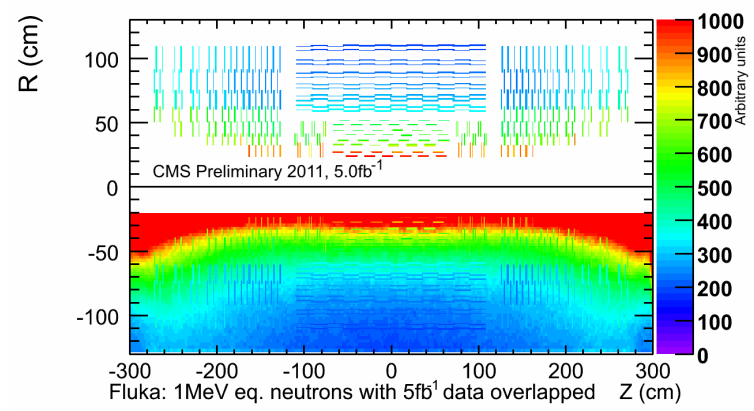

Figure 4: Comparing leakage current measurements (top, strip tracker only, corrected for sensor temperature) to FLUKA simulations (bottom, overlaid with leakage current measurements from top).

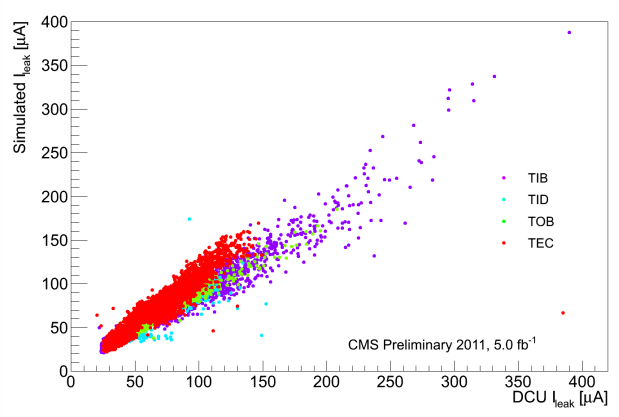

Figure 5: Plot of leakage current compared with simulation. Results are corrected for module type, radius, and sensor temperature.

\section{Hit reconstruction}

The first step towards high quality tracking data is the efficient and accurate reconstruction of hits from crossing particles. In figure 6 an example from the strip tracker of the signal-to-noise ratio for hits on reconstructed tracks is shown. The ratio is corrected for the angle of the track with respect to the sensor surface to correct for a possible difference in path length. A fit to the distribution with a Landau distribution convoluted with a Gaussian yields a most probable value (MPV) of 18.4 which allows for an efficient hit reconstruction.

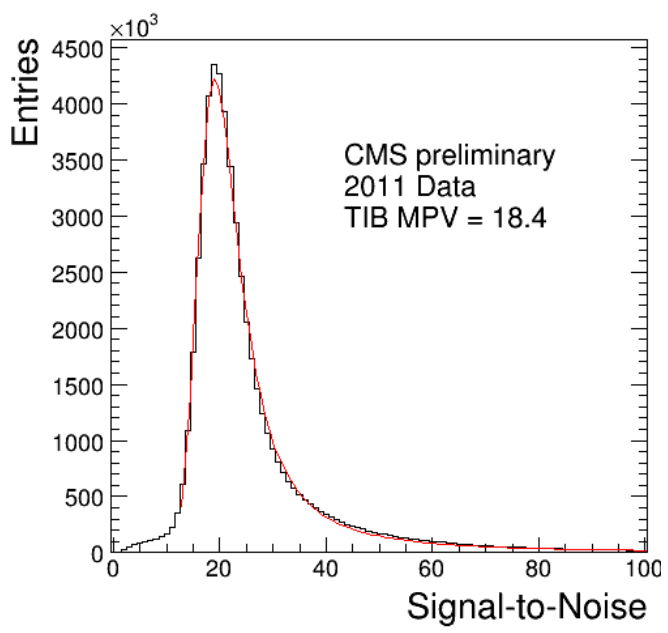

Figure 6: Exemplary signal-to-noise distribution from hits on reconstructed particle tracks. The ratio is corrected for the incident angle of the track on the module under investigation.

In figure $7 \mathrm{a}$ the resulting hit efficiency is shown for two cases. In black, the efficiency is shown in case one assumes a perfect detector without any non-functional modules. In red, the hit efficiency is shown when taking into account known inefficient regions. One can see that in this second case, the hit efficiency is well above $99 \%$ in all detector parts. In figure $7 \mathrm{~b}$ the hit efficiency 


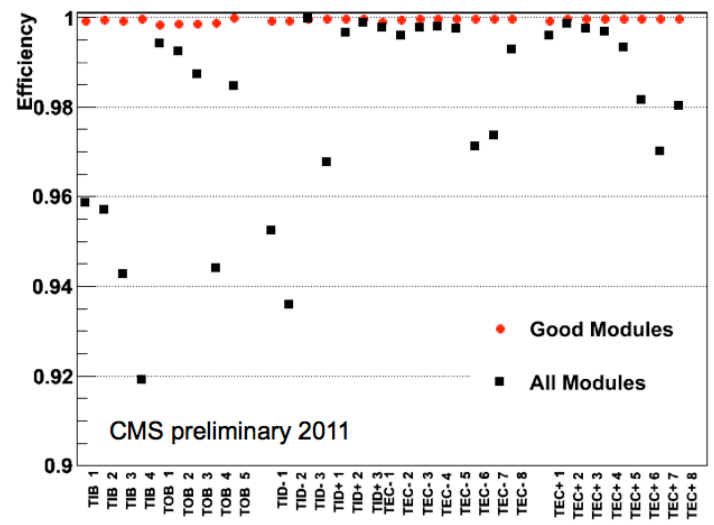

(a) Strip Tracker

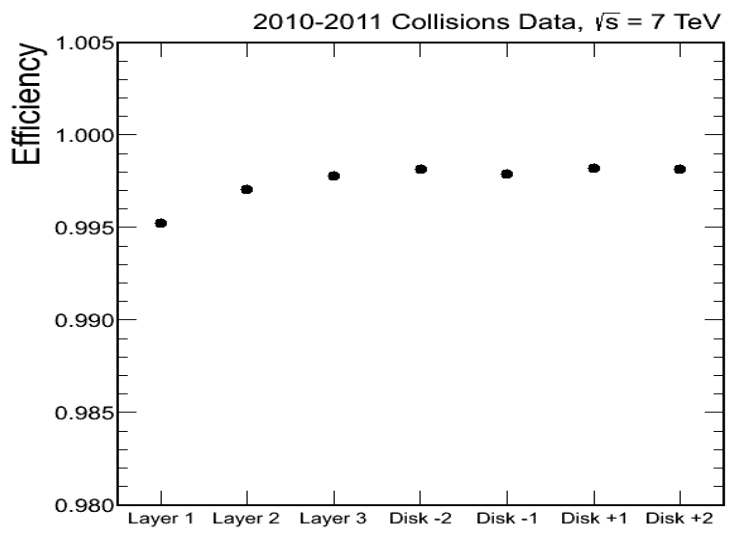

(b) Pixel Detector

Figure 7: Single hit efficiencies are measured in data from (a) the strip tracker and (b) pixel tracker, respectively. See main text for details.

for the pixel detector is shown. One can see a slight reduction of the efficiency for the innermost layers. This is an effect that depends on the instantaneous luminosity and the L1 trigger rate. It is discussed in detail in [6].

\section{Tracking}

CMS uses a multi-stage iterative approach for track reconstruction. Subsequent steps in the reconstruction process allow for looser requirements for track seeds and constraints with respect to the interaction region. Particle hits which have been assigned to a track are removed from latter iterations to reduce combinatorics. This allows for the reconstruction of more complicated track topologies with highly displaced vertices, conversions as well as lower $p_{\mathrm{T}}$ thresholds. In addition, later iterations recover potential inefficiencies from earlier iterations. Seeds for track reconstructions are reconstructed from a number of different detector patterns like triplets from pixel hits, mixed pairs (pixel or strip hits) together with the primary vertex, mixed triplets (pixel and strip hits) and others. The main tracking algorithm is based on pixel seeds and uses a Kalman filter.

In figure 8 the tracking efficiency from simulation is shown for muons as a function of the pseudorapidity. Three different bins of $p_{\mathrm{T}}$ are shown. The efficiency is largely flat and close to 99\% over the full $\eta$ coverage of the silicon tracker. In figure 9 the same efficiency is shown for charged pions and as function of $p_{\mathrm{T}}$. One can see that in the central region of the detector $(|\eta|<0.9)$ the effiency is well above $90 \%$ already for tracks of about $1 \mathrm{GeV}$. The efficiency drops slightly for tracks in the transition region $(0.9<|\eta|<1.4)$ and is lowest, yet still well above $80 \%$, in the endcap region $(|\eta|>1.4)$.

\section{Vertexing}

Another prime ingredient in the reconstruction of $p p$ collision events is the efficient and precise 


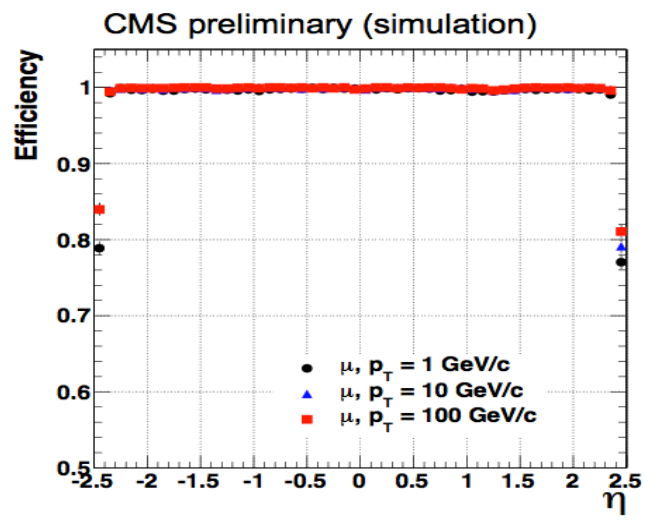

Figure 8: Muon reconstruction efficiency from simulation as function of $\eta$ for three different regions of $p_{\mathrm{T}}$.

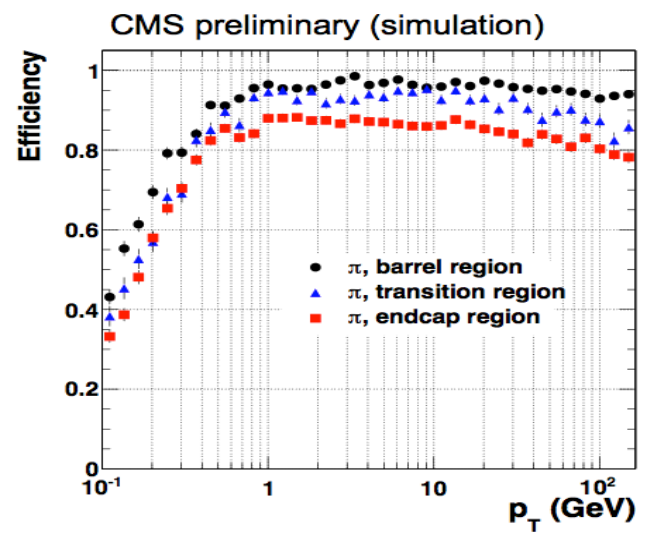

Figure 9: Pion reconstruction efficiency from simulation as function of $p_{\mathrm{T}}$ for three different regions of $\eta$.

reconstruction of primary and secondary vertices. To measure the efficiency and resolution, a socalled split method is used [6]. For this, tracks from one vertex from $p p$ collision events are divided into two subsets with roughly equal $p_{\mathrm{T}}$ distribution. One set of tracks is used as a tag whereas the second set serves as probe. To establish the vertex resolution, the two groups of tracks are used to reconstruct two vertices. The distribution of position differences between the two reconstructed vertices for a given number of tracks is fitted with a single Gaussian distribution and its mean value divided by $\sqrt{2}$ is taken as vertex resolution.

In figure 10 the vertex reconstruction efficiency is shown. One can see that it is very close to $100 \%$ already for two tracks with a $p_{\mathrm{T}}$ above $0.5 \mathrm{GeV}$ attached to it. The vertex resolution is shown in figure 11. It approaches 20(30) $\mu \mathrm{m}$ in $\mathrm{x}$ and $\mathrm{y}(\mathrm{z})$ for high number of tracks.

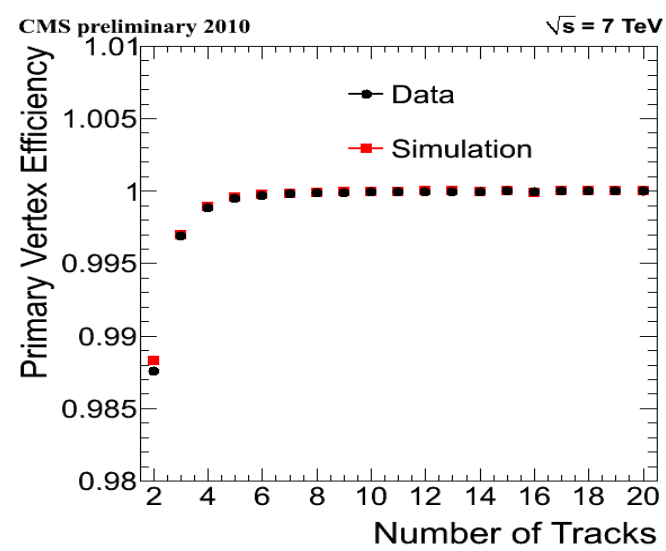

Figure 10: Primary vertex efficiency as function of number of tracks attached to the vertex for data (black) and simulation (red) [5].

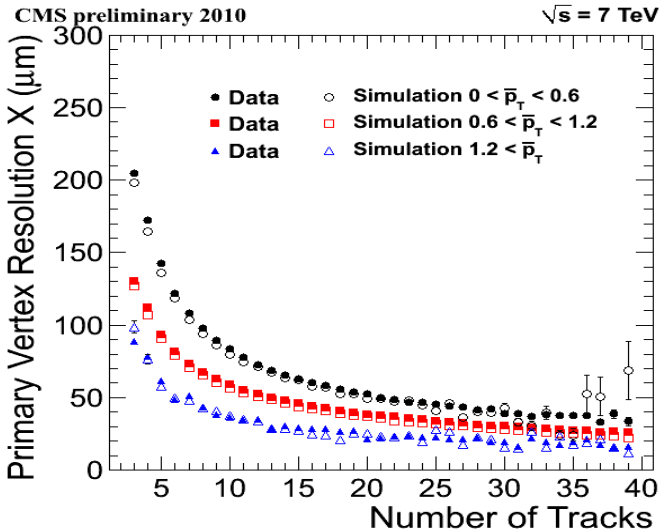

Figure 11: Primary vertex resolution as function of the number of tracks attached to the vertex for data (filled symbols) compared to simulation (open symbols) for different track $p_{\mathrm{T}}$ bins [5]. 


\section{Summary and Outlook}

The CMS silicon tracking sub-detectors were efficiently taking physics data during the 201012 LHC running with high uptime and excellent data quality. Hit reconstruction and tracking are working with high efficiency. Primary and secondary vertex reconstruction are also providing results with with supreme quality and very good efficiency. The integrated radiation dose of the detectors is continuously increasing and several methods are employed to closely monitor the evolution of the effects of radiation damage over time.

\section{References}

[1] S. Chatrchyan et al. [CMS Collaboration], "The CMS experiment at the CERN LHC," JINST 3 (2008) S08004.

[2] S. Paoletti et al., "The powering scheme of the CMS silicon strip tracker", in 10th Workshop on Electronics for LHC and future experiments, CERN-2004-010, CERN-LHCC-2004-030, http://cdsweb.cern.ch/record/814088.

[3] A. Ferrari, P. R. Sala, A. Fasso and J. Ranft, "FLUKA: A multi-particle transport code (Program version 2005)," CERN-2005-010.

[4] G. Battistoni, S. Muraro, P. R. Sala, F. Cerutti, A. Ferrari, S. Roesler, A. Fasso and J. Ranft, "The FLUKA code: Description and benchmarking,” AIP Conf. Proc. 896 (2007) 31.

[5] [CMS Collaboration], "Tracking and Primary Vertex Results in First 7 TeV Collisions," CMS-PAS-TRK-10-005.

[6] S. Chatrchyan et al. [CMS Collaboration], "Description and Performance of the CMS Track and Primary Vertex Reconstruction," to be submitted to JINST. 